\title{
La medicina rigenerativa in ortopedia e traumatologia
}

\author{
Achille Pellegrino \\ U.O.C. di Orto-Traumatologia, Ospedale "S.G. Moscati", Aversa (CE), Italia \\ achpell@tin.it
}

Pubblicato online: 29 ottobre 2019

(c) Società Italiana Ortopedici Traumatologi Ospedalieri d'Italia 2019

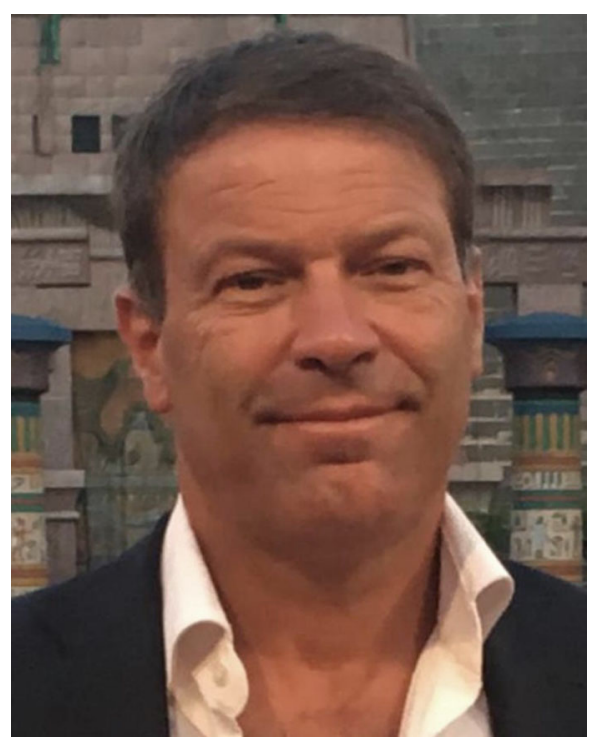

La Medicina Rigenerativa (MR) è una scienza che nasce pochi decenni fa, insieme ai concetti di "cellula staminale" e di "riparazione tissutale", apportando nuova linfa ed entusiasmo nella cura di diverse patologie di interesse ortopedico-traumatologico come, ad esempio, le malattie cronico-degenerative della cartilagine articolare o le patologie traumatiche, ovvero patologie che rivestono forte impatto sociale e influenti in modo significativo sulla qualità della vita.

Si definisce MR il processo di riparazione, di rigenerazione e di sostituzione di cellule, tessuti e organi umani, per ripristinare funzionalità fisiologiche compromesse da cause quali difetti congeniti, malattie, traumi o invecchiamento.

La rigenerazione dei tessuti può essere utilizzata con scopi diversi e in momenti diversi: prevenire la degenerazione, accelerare i tempi di guarigione e risolvere eventuali problemi postoperatori.

In particolare:

- prevenzione: le infiltrazioni di prodotti a base di cellule autologhe possono essere effettuate con intenzioni conserva- tive, cioè, per risolvere i sintomi di dolore e la diminuita funzionalità articolare

- accelerazione dei tempi di guarigione: l'utilizzo della medicina rigenerativa, combinato con l'intervento chirurgico, può migliorare in maniera significativa il risultato dell'intervento; accelera i tempi di guarigione e riduce il dolore postoperatorio

- risoluzione problematiche postoperatorie: la medicina rigenerativa può intervenire anche in ausilio a un intervento chirurgico che ha avuto un esito non completamente soddisfacente a causa di fattori che ostacolano la rigenerazione dei tessuti.

Uno dei settori più interessanti della MR, per le prospettive di impiego futuro, è l'utilizzo di cellule staminali mesenchimali (MSC) le quali, una volta espanse e differenziate, consentirebbero di riparare, in toto o in parte, il tessuto danneggiato, anche con semplice infusione a distanza dal tessuto bersaglio. Tali MSC costituiscono, pertanto, la prospettiva più futuribile in $\mathrm{MR}$, da sole ovvero in associazione a biomateriali che fungano da scaffold.

La MR si occupa dello sviluppo di terapie innovative e avanzate mirate alla ricostruzione di tessuti e organi irrimediabilmente danneggiati. L'integrità e la riparazione dei tessuti dipendono da una popolazione di cellule staminali adulte presente nei tessuti stessi e in grado di autorinnovarsi. Il trattamento con cellule staminali mesenchimali migliora in maniera significativa la guarigione, diminuisce il dolore postchirurgico e accelera il recupero e la riabilitazione sia per quanto concerne la vita sociale e lavorativa, sia per l'eventuale ripresa di attività sportiva. L'efficacia, la maneggevolezza del trattamento, la mini-invasività e la sostanziale assenza di effetti collaterali e/o reazioni avverse rende, inoltre, tale trattamento un'opzione futuristica e complementare ai trattamenti farmacologici e chirurgici, laddove non sia addirittura sostitutiva degli stessi. In tale contesto, il ruolo del medico risulta cruciale: è suo il compito di amalgamare in- 
sieme le varie tecnologie per l'ottenimento del miglior risultato clinico, così come la collaborazione tra specialisti di vari settori è fondamentale nella selezione dei pazienti, nel monitoraggio clinico degli stessi e, quindi, nell'uso corretto del "concentrato" di tessuti, ricchi di cellule staminali e di altri componenti, compresi i fattori di crescita.

In questo fascicolo monotematico, affidato all'ACOTO, con la collaborazione di valenti colleghi appartenenti ad altre Società regionali, sono state affrontate le diverse problematiche e i numerosi interrogativi della MR, raccogliendo le esperienze di ciascuno, con l'intento di giungere a indicazioni terapeutiche e a protocolli di trattamento univoci, col fine comune del benessere dei pazienti.

Un sincero e appassionato "grazie" va a tutti coloro i quali, con provata capacità e con grande esperienza ma, soprattutto, con la passione che contraddistingue il nostro lavoro e la nostra mission, hanno fornito un valido contributo alla stesura di codesto volume scientifico. Un ringraziamento particolare va anche all'OTODI che ha accolto la nostra proposta di curare codesto fascicolo monotematico, nonché ai Direttori Scientifici de Lo Scalpello per il prezioso supporto fornito nell'elaborazione dei manoscritti. 